\title{
1 A Dual-reporter Platform for Screening Tumor-targeted Extracellular Vesicles
}

2

3 Masamitsu Kanada*1,2, Lauren Linenfelser ${ }^{1,3}$, Elyssa Cox ${ }^{1}$, Assaf A. Gilad ${ }^{* 1,3,4}$

4

\section{Affiliations}

$6 \quad{ }^{1}$ Institute for Quantitative Health Science and Engineering (IQ), ${ }^{2}$ Department of Pharmacology \&

7 Toxicology, ${ }^{3}$ Department of Biomedical Engineering, ${ }^{4}$ Department of Radiology, Michigan State

8 University, East Lansing, MI, United States.

9

$10 *$ Corresponding authors:

11 Masamitsu Kanada, Michigan State University, 775 Woodlot Dr. East Lansing, MI 48824. Phone:

12 (517) 884-6933; E-mail: kanadama@msu.edu

13 Assaf A. Gilad, Michigan State University, 775 Woodlot Dr. East Lansing, MI 48824. Phone:

14 (517) 884-7468; E-mail: gilad@msu.edu

15

16 Keywords: extracellular vesicles, bioluminescence resonance energy transfer, tumor homing

17 peptide, lipid nanoprobes, fluorescence microscopy, breast cancer 


\section{Abstract}

20 Extracellular vesicle (EV)-mediated transfer of biomolecules plays an essential role in intercellular

21 communication and may improve targeted drug delivery. In the past decade, various approaches

22 to EV surface modification for targeting specific cells or tissues have been proposed, including 23 genetic engineering of parental cells or postproduction EV engineering. However, due to technical

24 limitations, targeting moieties of engineered EVs have not been thoroughly characterized. Here, 25 we report the bioluminescence resonance energy transfer (BRET) EV reporter, PalmReNL-based 26 dual-reporter platform for characterizing the cellular uptake of tumor homing peptide (THP)27 engineered EVs, targeting PDL1, uPAR, or EGFR proteins expressed in MDA-MB-231 breast 28 cancer cells, simultaneously by bioluminescence measurement and fluorescence microscopy. 29 Bioluminescence analysis of cellular EV uptake revealed the highest binding efficiency of uPAR30 targeted EVs, whereas PDL1-targeted EVs showed slower cellular uptake. EVs engineered with 31 two known EGFR-binding peptides via lipid nanoprobes did not increase cellular uptake, 32 indicating that designs of EGFR-binding peptide conjugation to the EV surface are critical for 33 functional EV engineering. Fluorescence analysis of cellular EV uptake allowed us to track 34 individual PalmReNL-EVs bearing THPs in recipient cells. These results demonstrate that the 35 PalmReNL-based EV assay platform can be a foundation for high-throughput screening of tumortargeted EVs. 


\section{Introduction}

Extracellular vesicles (EVs) provide a natural delivery system that transfers various cellular cargo

41 (protein, mRNA, non-coding RNA, and DNA) to both adjacent and distant cells in the body [1].

42 EV-based drug delivery has been an active research field in the past decade [2], and previous preclinical studies demonstrated the superiority of EV-based drug delivery over delivery using synthetic nanocarriers, such as liposomes [3, 4].

EVs are heterogeneous populations of nanoscale membrane-enclosed vesicles released virtually from all cell types. At least two main EV classes are released from mammalian cells in the physiological condition: exosomes ( $\sim 30-120 \mathrm{~nm}$ in diameter), formed by inward budding of the endosomal membrane, producing multivesicular bodies (MVBs) that fuse with the plasma membrane [5]; microvesicles (50-1000 $\mathrm{nm}$ in diameter), formed by outward budding at the plasma membrane [1]. EVs offer advantages for surface engineering while possessing inherent immune evasion and tissue penetrating characteristics $[3,6,7]$. Several approaches have achieved targeted delivery of engineered EVs using various targeting moieties, including antibodies, nanobodies, and peptides [8]. As shown in pioneering studies, EVs have been modified with tissue-targeting peptides by genetically engineering the parental cells - for example, Lamp2b was fused to neuronspecific rabies viral glycoprotein (RVG) peptides [9] or angiogenic vasculature-specific iRGD peptides [10]. Similarly, EGFR-targeting GE11 peptides were genetically fused to the transmembrane domain of the platelet-derived growth factor receptor [11]. As alternative approaches for functionalizing the EV surface with peptides, both covalent and noncovalent postproduction modifications have been proposed - for example, the cyclo(Arg-Gly-Asp-D-TyrLys) peptides were coupled to the EV surface through bio-orthogonal copper-free azide alkyne cycloaddition for targeting ischemic reactive cerebral vascular endothelial cells [12]. Moreover, a

62 recent study demonstrated the covalent conjugation of EVs with high copy numbers of GE11

63 peptides using protein ligases for targeting EGFR-positive lung cancer cells both in vitro and in

64 vivo [13]. On the other hand, various approaches for noncovalent postproduction modification of

65 EVs were succeeded. For example, EVs were engineered with stearylated artificial leucine zipper

$66 \mathrm{~K} 4$ peptides or arginine-rich cell-penetrating peptides (CPPs) to induce active macropinocytosis

67 in vitro [14, 15]. More recently, cardiac homing peptide (CHP)-coupled lipid nanoprobes were

68 inserted into the EV membrane for targeting the infarcted heart in murine models [16, 17]. 
Despite the recent advances in the field, a sensitive and quantitative assay platform for screening engineered EVs is still lacking. Lipophilic fluorescent dyes such as PKH, DiR, and DiI

71 are often used to assess the cellular uptake of EVs due to their simple labeling method [18]. While

72 their bright fluorescence may be advantageous for visualizing individual EVs, quantitative 73 assessment of cellular uptake of labeled EVs by bulk measurement (e.g., plate readers) is still a 74 challenge due to the background autofluorescence in culture media. Alternatively, bioluminescent 75 reporters (Gaussia luciferase [Gluc] [19, 20] and NanoLuc [21, 22]) have been developed for 76 assessing in vivo biodistribution, EV release, and cellular EV uptake. Moreover, a recent study 77 demonstrated a bioluminescence resonance energy transfer (BRET)-based EV reporter, 78 PalmGRET [23], for multiresolution imaging of reporter EVs. PalmGRET consists of an eGFP79 NanoLuc fusion reporter protein (GpNluc [24]) and a palmitoylation signal sequence [25]. Notably, because of the acid-quenching mechanism [26], the most widely used eGFP is acidic pH-sensitive (pKa 6.0). Since recent studies revealed that endocytosed EVs were rapidly translocated into endosomal compartments [27, 28], acidic $\mathrm{pH}$-sensitive eGFP (pKa 6.0) is not an ideal reporter for tracking cellular EV uptake. Therefore, in this study, we used a novel BRET EV reporter, PalmReNL [29], consisting of a tdTomato-NanoLuc fusion protein [30] (pKa for tdTomato: 4.7) and a palmitoylation signal sequence [25], to characterize cellular EV uptake as a dual-reporter platform. We developed tumor-targeted EVs using several tumor homing peptides (THPs) [31] coupled to lipid nanoprobes for noncovalent postproduction EV engineering and assessed their increased capability of cancer cell targeting by bioluminescence measurement and fluorescence microscopy in vitro. The PalmReNL-based assay system provides a foundation for highthroughput screening of engineered EVs for tumor-targeted drug delivery.

\section{Materials and Methods}

\section{Plasmid DNA constructs}

94 For the EV reporter, PalmReNL, a palmitoylation sequence (MLCCMRRTKQ) [25, 32] was 95 genetically fused to Red enhanced Nano-lantern (ReNL [30]; Addgene plasmid \#89536, gift from 96 Takeharu Nagai) by PCR and subcloned into the Sleeping Beauty transposon vector [33] as we 97 previously reported [29]. HEK293FT cells were cotransfected with a plasmid encoding PalmReNL 98 and pCMV(CAT)T7-SB100 (Addgene \#34879, gift from Zsuzsanna Izsvak). 


\section{Cell culture}

MDA-MB-231 cells (ATCC) and HEK293FT cells (R700-07, Invitrogen) were cultured in Dulbecco's modified eagle medium supplemented with GlutaMax (Gibco), 10\% (vol/vol) FBS and $1 \%$ penicillin/streptomycin and incubated at $37{ }^{\circ} \mathrm{C}$ in a $5 \% \mathrm{CO}_{2}$ atmosphere. HEK293FT cells stably expressing PalmReNL were selected in the presence of puromycin $(2 \mu \mathrm{g} / \mathrm{mL})$ to establish cells constitutively expressing PalmReNL. We further enriched cells highly expressing PalmReNL by limiting dilution.

\section{EV isolation}

EV-depleted FBS was prepared by 18 -h ultracentrifugation at 100,000 g, $4{ }^{\circ} \mathrm{C}$ [34]. PalmReNLHEK293FT cells were seeded at $1.5 \times 10^{6}$ cells per $100-\mathrm{mm}$ cell culture dish and cultured for 2-3 $\mathrm{d}$ in $10 \mathrm{~mL}$ of EV-depleted media. The conditioned medium from a $10 \mathrm{~mm}$ cell culture dish was centrifuged at $600 \mathrm{~g}$ for $15 \mathrm{~min}$ to remove cells and debris. The supernatant was centrifuged at $2,000 \mathrm{~g}$ for $20 \mathrm{~min}$ at room temperature (RT) to remove apoptotic bodies. The supernatant was filtered through $0.2 \mu \mathrm{m}$ PES membrane filter (Nalgene, 725-2520) with pressure to remove large vesicles. EVs were collected by a size-based EV isolation method [35, 36] using $50 \mathrm{~nm}$ membrane filter (Whatman, WHA110603) with holders (EMD Millipore, SX0002500). Holders with $50 \mathrm{~nm}$ membrane filters were connected to a vacuum manifold (Qiagen) and washed with $10 \mathrm{~mL}$ of PBS by applying vacuum. The remaining EVs in the supernatant were trapped on the membranes. Then, the enriched EVs were washed with $5 \mathrm{~mL}$ of PBS by applying vacuum and 500-1000 $\mu \mathrm{L}$ of samples were carefully collected. All EVs were aliquoted and stored at $-80^{\circ} \mathrm{C}$.

\section{Nanoparticle tracking analysis (NTA)}

EVs derived from PalmReNL-HEK293FT cells were analyzed using the ZetaView Multiple Parameter Particle Tracking Analyzer (Particle Metrix) following the manufacturer's instructions. The enriched PalmReNL-EVs were diluted 100- to 1,000-fold with PBS for the measurement of particle size and concentration.

\section{EV membrane modification}

PalmReNL-EVs were engineered with peptides known as THPs (a uPAR-binding peptide: VSNKYFSNIHWGC [37]; a PDL1-binding peptide: YASYHCWCWRDPGRS [38, 39]; an 
131 EGFR-binding peptide (KKKGG-GE11): KKKGGYHWYGYTPQNVI [40, 41]; and an EGFR-

132 binding peptide \#2: YHWYGYTPENVI [42]). DOPE-NHS (dioleoylphosphoethanolamine N-

133 hydroxysuccinimide; COATSOME FE-8181SU5, NOF America, White Plains, NY) was coupled

134 to synthesized THPs (GenScript, Piscataway, NJ) for self-insertion of the THP-lipid nanoprobes

135 into EV membranes as previously reported with slight modifications [17]. The THP and DOPE-

136 NHS were dissolved in DMSO at $1 \mathrm{mg} / \mathrm{mL}$ and $0.25 \mathrm{mg} / \mathrm{mL}$, respectively. The DOPE-NHS and

137 THP were combined with a 100-fold molar excess of THPs in the presence of an equal vol of

138 HEPES buffer (25 mM) - 1.3 nmol DOPE-NHS plus $130 \mathrm{nmol}$ THPs with $12.5 \mathrm{mM}$ HEPES-

139 and allowed to react at RT for $1 \mathrm{~h}$ to form the DOPE-peptides. 10\% (vol/vol) Tris buffer (100 mM)

140 was added to stop the reaction. A negative control, DOPE-lipid nanoprobe only, was prepared in

141 the same condition without THPs. PalmReNL-EVs $\left(1.5 \times 10^{9}\right)$ were then added and incubated with

142 DOPE-THPs or DOPE only at $37^{\circ} \mathrm{C}$ for $30 \mathrm{~min}$. The engineered EVs were concentrated using 100

$143 \mathrm{kDa}$ ultrafiltration spin filter (UFC810024, MilliporeSigma) and washed with PBS twice by

144 centrifugation. The engineered EVs in $250 \mu \mathrm{L}$ were stored at $-80{ }^{\circ} \mathrm{C}$.

Fluorescence microscopy and bioluminescence measurements

147 The uptake of PalmReNL-EVs by MDA-MB-231 cells was analyzed by fluorescence microscopy

148 and bioluminescence measurement. The cells were plated in 96-well black/clear bottom cell

149 culture plates at a concentration of 45,000 cells/well and cultured for $24 \mathrm{~h}$. The culture medium

150 was replaced with EV-depleted medium and the THP-engineered PalmReNL-EVs were added at

151 a concentration of $6.0 \times 10^{8}$ or $6.0 \times 10^{9} \mathrm{EVs} / \mathrm{mL}$. At the end of the incubation period, the uptake

152 of the reporter was analyzed by measuring bioluminescence after adding furimazine (Fz; $25 \mu \mathrm{M})$

153 using a Spark Multimode Microplate Reader (Tecan, Männedorf, Switzerland). Phase contrast and

154 fluorescence images of PalmReNL-HEK293FT cells, PalmReNL-EVs, or MDA-MB-231 cells

155 treated with the reporter EVs were taken using All-in-one Fluorescence Microscope BZ-X700

156 (KEYENCE). For assessing cellular uptake of PalmReNL-EVs, the cells in a glass bottom chamber

157 slide ( $\mu$-Side 8 Well Glass Bottom, ibidi) were stained with $10 \mu \mathrm{g} / \mathrm{mL}$ Hoechst 33342 (H3570,

158 Life Technologies) before fluorescence microscopy was performed using DeltaVision Microscope

159 (GE Healthcare Life Sciences). All images were further analyzed using the ImageJ software

160 (imagej.nih.gov). 


\section{Western blotting}

163 Whole cell lysates and EVs $\left(4 \times 10^{8}\right.$ EVs $)$ were derived from PalmReNL-HEK293FT cells and 164 mixed with $4 \times$ sample buffer (Bio-Rad) with $\beta$-mercaptoethanol for detecting Alix, CD9, TSG101, 165 and tdTomato proteins. Whole cell lysates derived from MDA-MB-231 cells were mixed with $2 \times$ sample buffer without $\beta$-mercaptoethanol (non-reducing conditions) for detecting PDL1, uPAR, EGFR, and GAPDH. Proteins were separated on a 4\%-20\% Mini-PROTEAN TGX gel (Bio-Rad) and transferred to a polyvinylidene difluoride membrane (Millipore, IPFL00010). After blocking with 5\% non-fat dry milk at RT for $1 \mathrm{~h}$, membranes were probed with primary antibodies overnight at $4{ }^{\circ} \mathrm{C}$ at dilutions recommended by the suppliers as follows: anti-Alix (Proteintech, 12422-1-AP), anti-TSG101 (Proteintech, 14497-1-AP), anti-CD9 (Proteintech, 60232-1-lg), anti-RFP (Rockland Immunochemicals, 600-401-379), anti-PDL1 (Proteintech, 66248-1-1g), anti-uPAR (Proteintech, 10286-1-AP), anti-EGFR (Proteintech, 18986-1-AP), or anti-GAPDH (Santa Cruz, G-9), followed by incubation with horseradish peroxidase (HRP)-conjugated secondary antibodies at RT for $1 \mathrm{~h}$.

175 The membranes were visualized with ECL select Western Blotting Detection Reagent (GE 176 Healthcare, RPN2235) on ChemiDoc MP Imaging System (Bio-Rad).

\section{Transmission electron microscopy}

179 The samples were prepared as previously reported with slight modifications [36, 43]. Isolated EVs

180 (PalmReNL-EVs with or without uPAR-binding peptides: $6.0 \times 10^{9} \mathrm{EVs} / \mathrm{mL}$ ) were fixed in $1 \%$ 181 paraformaldehyde at RT for $30 \mathrm{~min}$. For negative-staining of EVs, $5 \mu \mathrm{L}$ of the sample solution 182 was placed on a carbon-coated EM grid, and EVs were immobilized for $1 \mathrm{~min}$. Next, the samples 183 were washed by placing each one face down on top of a $100 \mu \mathrm{L}$ droplet of water $(6 \times)$. The samples 184 were negative stained with 1\% uranyl acetate. Excess uranyl acetate was removed by contacting 185 the grid edge with filter paper and the grid was air-dried. Samples were observed using a JEOL 1861400 Flash Transmission Electron Microscope equipped with an integrated Matataki Flash 187 sCMOS bottom-mounted camera. The 1400 Flash was operated at $100 \mathrm{kV}$. 
All data are presented as mean \pm SD. Statistical analysis was performed using Prism 9 (GraphPad Software). A two-tailed Student's $t$-test was used for comparisons between two groups. A value of $p<0.05$ was considered significant and is indicated on all figures with an asterisk.

\section{Results}

Characterization of dual-reporter EVs derived from HEK293FT cells stably expressing PalmReNL.

We have previously utilized PalmReNL [29] as a dual-reporter for bioluminescence and fluorescence tracking of EVs in vitro and in vivo. In the current study, we generated HEK293FT cells stably expressing PalmReNL and selected the EV donor cells that maintained a high level of PalmReNL expression (Figure 1A, B). We next isolated PalmReNL-EVs from the conditioned medium using $50 \mathrm{~nm}$ membrane filters as we previously reported [35, 36] and characterized them by nanoparticle tracking analysis (NTA). The concentration of PalmReNL-EVs was $1.0 \times 10^{10}$ $\mathrm{EVs} / \mathrm{mL}$ and their mean diameter was $117.6 \mathrm{~nm}$ (Figure 1C). The protein concentration of $1.0 \times$ $10^{10} \mathrm{EVs} / \mathrm{mL}$ PalmReNL-EVs was $557 \mu \mathrm{g} / \mathrm{mL}$. Western blotting analysis of exosome markers, including Alix, CD9, and TSG101, in the whole-cell lysate and isolated PalmReNL-EVs, confirmed successful EV enrichment in our experimental setting (Figure 1D). In addition, tdTomato proteins were detected in both the whole-cell lysate and PalmReNL-EVs.

Next, we measured bioluminescence signals in different concentrations of PalmReNL-EVs using furimazine $(25 \mu \mathrm{M})$ as the substrate (Figure 1E). The bioluminescence signals were proportional to the concentrations of PalmReNL-EVs, which allows us to assess relative EV amounts by measuring bioluminescence. Moreover, individual PalmReNL-EVs were visualized by exciting tdTomato (Figure 1F) as ReNL is a fusion protein of tdTomato and NanoLuc [30]. These results demonstrated that PalmReNL-EVs allow for rapid quantification of isolated reporter EVs as well as cellular EV uptake in vitro. Moreover, PalmReNL can be used as a fluorescent EV reporter for standard microscopy and flow cytometry as well.

\section{Engineering the surface of PalmReNL-EVs with tumor homing peptides (THPs).}

Engineering the surface of EVs with tumor-targeting ligands has recently shown significant success by improving the tumor cell-binding capability of EVs [10]. Recent studies have developed a lipid nanoprobe system for rapidly isolating EVs [44] and directing therapeutic EVs 
221 to the infarcted heart in animal models [16, 17]. In this study, we engineered PalmReNL-EVs with

222 well-characterized three THPs, which bind Urokinase-type plasminogen activator receptor (uPAR),

223 Programmed death ligand 1 (PDL1), or Epidermal growth factor receptor (EGFR) (Figure 2A).

224 First, we characterized the peptide-engineered PalmReNL-EVs by transmission electron 225 microscopy (TEM) (Figure 2B). A heterogeneous mixture of PalmReNL-EVs showed artifactual 226 cup-shaped morphology [45]. There was no noticeable morphological change in the EVs 227 engineered with the uPAR-binding peptide-coupled lipid nanoprobes compared to PalmReNL228 EVs without membrane modification (Figure 2B).

229 The peptide-engineered PalmReNL-EVs were tested on MDA-MB-231 breast cancer cells 230 in vitro. MDA-MB-231 cells display a mesenchymal-like phenotype representing a good model 231 for metastatic breast cancer [46] (Figure 2C). Previous studies reported that three target molecules, 232 PDL1 [39], uPAR [37], and EGFR [42], were highly expressed in human breast cancer cells. 233 Before carrying out binding assays, we confirmed the high protein expressions of PDL1, uPAR, 234 and EGFR in MDA-MB-231 cells by western blotting (Figure 2D). All the target membrane 235 proteins were highly expressed in MDA-MB-231 cells, indicating that they are ideal targets for 236 characterizing tumor-targeted EVs.

Bioluminescence analysis of cellular uptake of THP-engineered PalmReNL-EVs by MDAMB-231 cells.

Cellular uptake of THP-engineered PalmReNL-EVs by MDA-MB-231 cells was assessed by measuring bioluminescence signals. Recipient cells were cultured with two different concentrations of PalmReNL-EVs $\left(6.0 \times 10^{8}\right.$ or $\left.6.0 \times 10^{9} \mathrm{EVs} / \mathrm{mL}\right)$ with or without peptides targeting uPAR, PDL1, or EGFR for $4 \mathrm{~h}$ before measuring bioluminescence (Figure 3A). A higher concentration $\left(6.0 \times 10^{9} \mathrm{EVs} / \mathrm{mL}\right)$ of PalmReNL-EVs bearing PDL1- or uPAR-binding peptides increased their uptake by MDA-MB-231 cells by 1.9- and 22.5-fold relative to PalmReNL-EVs engineered with DOPE-lipid nanoprobe only, whereas a lower concentration $\left(6.0 \times 10^{8} \mathrm{EVs} / \mathrm{mL}\right)$ increased by 2.3- and 8.1-fold, respectively (Figure 3A). This result demonstrated that a higher EV concentration was better for testing PalmReNL-EVs bearing uPAR-binding peptides with higher cellular uptake efficiency. On the other hand, the two EV concentrations tested did not affect the relative cellular uptake efficiency of PalmReNL-EVs bearing PDL1-binding peptides compared to PalmReNL-EVs with DOPE-lipid nanoprobe only. 
Of note, PalmReNL-EVs engineered with the EGFR-binding peptide GE11 [40] did not

253

254

255

256

257

258

259

260

261

262

263

264

265

266

267

268

269

270

271

272

273

274

275

276

277

278

279

280

281

282 significantly increase their cellular uptake in our experimental conditions. We hypothesized that the affinity of GE11 peptides might be reduced by inserting into the EV membrane. To test this possibility, we assessed PalmReNL-EVs engineered with another EGFR-binding peptide sequence that previously showed the improved EGFR-binding efficiency [42]. However, PalmReNL-EVs engineered with the EGFR-binding peptide \#2 did not increase their uptake by MDA-MB-231 cells (Figure 3B). This result suggests that engineering EVs with these EGFR-binding peptides via DOPE-lipid nanoprobes may disturb their binding with EGFR proteins on the surface of cancer cells or may not have sufficient stability of the EGFR-binding peptides on the EV surface.

\section{Bioluminescence analysis of time-dependent cellular uptake of THP-engineered PalmReNL-} EVs.

We next evaluated cellular uptake of THP-engineered PalmReNL-EVs by MDA-MB-231 at various time points by measuring bioluminescence signals. The recipient cells were cultured with PalmReNL-EVs $\left(6.0 \times 10^{9} \mathrm{EVs} / \mathrm{mL}\right)$ bearing THPs or DOPE-lipid nanoprobes only for 4, 8, 16, and $24 \mathrm{~h}$ before bioluminescence analysis. Interestingly, MDA-MB-231 cells cultured with PalmReNL-EVs engineered with uPAR-binding peptides showed a rapid decrease of bioluminescence signals-22.1-, 11.3-, 5.9-, and 7.57-fold higher bioluminescence signals at 4, 8, 16, and 24 h, respectively (Figure 4B), relative to PalmReNL-EVs with DOPE-lipid nanoprobes only (Figure 4A). On the other hand, PalmReNL-EVs bearing PDL1-binding peptides slowly increased bioluminescence signals over time-1.34-, 2.2-, 3.0-, and 1.89-fold higher bioluminescence signals at 4, 8, 16, and $24 \mathrm{~h}$, respectively (Figure 4C), relative to PelmReNL-EVs with DOPE-lipid nanoprobes only (Figure 4A). PalmReNL-EVs bearing EGFR-binding peptides (GE11) did not show a significant increase in their uptake by MDA-MB-231 cells even after longer culture periods (Figure 4D).

A previous study demonstrated the loss of NanoLuc enzyme activity with endosomal translocation [47]. We also found that PalmReNL-EVs decreased their activity at pH below 6.0 either with or without detergent treatment [29]. Thus, the signal loss of PalmReNL-EVs bearing uPAR-binding peptides with higher binding efficiency at long culture periods may be due to the acidic pH sensitivity of PalmReNL in endosomal compartments. However, PalmReNL-EVs bearing PDL1-binding peptides slowly increased the bioluminescence signal in the recipient cells 
over the long culture periods, possibly due to continuous cellular uptake of PalmReNL-EVs bearing PDL1-binding peptides with lower binding efficiency.

\section{Fluorescence analysis of cellular uptake of THP-engineered PalmReNL-EVs.}

287 We further characterized cellular uptake of PalmReNL-EVs bearing uPAR-, PDL1-, or EGFRbinding peptides by assessing tdTomato fluorescence (Figure 5). Importantly, in PalmReNL-EVs, NanoLuc enzyme activity and tdTomato fluorescence property exhibit different acidic $\mathrm{pH}$ sensitivities $[48,49]$. While most fluorescent proteins are sensitive to acidic cellular environments such as late endosomes ( $\mathrm{pH}$ 5.5-6.0) and lysosomes ( $\mathrm{pH} 4.5-5.5)$, tdTomato fluorescence ( $\mathrm{pKa} 4.7)$ is less sensitive to acidic pH than eGFP ( $\mathrm{pKa}$ 6.0) [49]. In addition, we previously revealed that PalmReNL-EVs retained better fluorescence signals as assessed by flow cytometry in the recipient cells at the $24 \mathrm{~h}$ culture period compared to bioluminescence signals [29].

We cultured MDA-MB-231 cells in EV-depleted media for 8, 16, and $24 \mathrm{~h}$ with PalmReNL-EVs $\left(6.0 \times 10^{9} \mathrm{EVs} / \mathrm{mL}\right)$ bearing THPs or DOPE-lipid nanoprobes only as a negative control and examined individual reporter EVs in the recipient cells by fluorescence microscopy. Punctate fluorescence signals were observed in the recipient cells cultured with PalmReNL-EVs bearing uPAR- and PDL1-binding peptides for $8 \mathrm{~h}$ (Figure 5C, D), compared to PalmReNL-EVs with DOPE-lipid nanoprobes only or EGFR-binding GE11 peptides (Figure 5A, B). Notably, we also detected similar punctate fluorescence signals in the recipient cells cultured with PalmReNLEVs bearing uPAR- and PDL1-binding peptides both at the $16 \mathrm{~h}$ and $24 \mathrm{~h}$ culture periods (Figure

5G, H, K, L), whereas MDA-MB-231 cells incubated with PalmReNL-EVs bearing DOPE-lipid nanoprobes only or EGFR-binding peptides showed no significant tdTomato fluorescence signals even after longer culture periods (Figure 5E, F, I, J).

\section{Discussion}

308 We have developed a PalmReNL-based dual-reporter platform for characterizing tumor-targeted 309 EVs by bioluminescence measurement and fluorescence microscopy. Bioluminescence 310 measurement enables highly sensitive and quantitative reporter EV detection with negligible 311 background signals. In addition, fluorescence analysis of PalmReNL-EVs enables us to track 312 individual engineered EVs in recipient cancer cells. Moreover, since tdTomato is less sensitive to 313 acidic $\mathrm{pH}$ relative to eGFP, PlamReNL may be a superior EV reporter for tracking individual 
314

315

316

317

318

319

320

321

322

323

324

325

326

327

328

329

330

331

332

333

334

335

336

337

338

339

340

341

342

343

344

reporter EVs in acidic endosomal compartments by standard fluorescence microscopy or flow cytometry.

Recent studies have demonstrated infarct-directed cardiosphere-derived EVs (CDC-EVs), using a CHP [50], to increase the delivery efficacy and decrease the effective dose of intravenously administered CDC-EVs to reduce undesirable off-target effects [16, 17]. The CHP-engineered CDC-EVs increased cellular uptake by cardiomyocytes in vitro, and the engineered EVs improved functional recovery in an ischemia/reperfusion rat model by reducing fibrosis, inducing cardiomyocyte proliferation, and promoting angiogenesis [17]. In the current study, we used the same lipid nanoprobe technique for postproduction engineering of EVs with THPs targeting PDL1, uPAR, and EGFR expressed in human breast cancer cells. Interestingly, we found that EVs engineered with these THPs showed distinct binding characteristics against MDA-MB-231 cells in vitro-PalmReNL-EVs engineered with uPAR-binding peptides showed faster and more efficient cellular uptake, whereas reporter EVs bearing PDL1-binding peptides showed slower and less efficient cellular uptake. While we tested two different EGFR-binding peptide sequences coupled to the DOPE-lipid nanoprobe, PalmReNL-EVs bearing either EGFR-binding peptides did not increase their uptake by MDA-MB-231 cells. Previous studies have demonstrated EV-based EGFR targeting by genetically fusing the transmembrane domain of platelet-derived growth factor receptor to GE11 peptides [11] or by the covalent postproduction modification of EVs with GE11 peptides using protein ligases [13]. Therefore, we concluded that designs of targeting peptide conjugation on the EV surface may be essential for creating functional EGFR-targeted EVs.

We previously revealed that bioluminescence signals in PalmReNL-EVs rapidly decreased after cellular uptake due to their sensitivity to acidic cellular compartments [29]. This unique aspect of PalmReNL allows us to distinguish between EV binding to the cell surface and their uptake by recipient cells, which is challenging to assess using fluorescent EV reporters in a highthroughput setting. In the current study, PalmReNL-EVs engineered with uPAR-binding peptides showed the highest bioluminescence signals at the earlier time point; however, their bioluminescence signals rapidly decreased over the longer culture periods (Figure 4B vs. Figure 4A). This result suggests that uPAR-targeted reporter EVs are rapidly taken up by MDA-MB-231 cells and traffic into the endosome pathway as recently reported on HT1080 fibrosarcoma cellderived EVs [27]. On the other hand, while PDL1-targeted PalmReNL-EVs showed less efficient cellular EV uptake at the earlier time point, their bioluminescence signals slowly increased even 
345 after the longer culture periods (see 8 and $16 \mathrm{~h}$ in Figure 4C vs Figure 4A), suggesting that PDL1-

346 targeted EVs may slowly bind to the cell surface. Since ReNL consists of tdTomato and NanoLuc,

347 we further followed individual THP-engineered PalmReNL-EVs in the recipient MDA-MB-231

348 cells by fluorescence microscopy. Importantly, we detected consistent punctate fluorescence

349 signals of THP-engineered PalmReNL in the recipient cells during the culture period we tested

350 (Figure 5), possibly since tdTomato is less sensitive to acidic endosomal compartments. While 351 quantitatively analyzing cellular uptake of uPAR-targeted and PDL1-targeted PalmReNL-EVs

352 based on their fluorescence signals is challenging, it allows for long-term tracking of individual 353 tumor-targeted reporter EVs in recipient cells.

\section{5. Conclusions}

356 We have developed a novel approach for screening THP-engineered EVs using PalmReNL as a 357 dual EV reporter for bioluminescence measurement and fluorescence microscopy in vitro. In this 358 study, three THPs targeting PDL1, uPAR, and EGFR were tested for postproduction engineering 359 of EVs via a DOPE-lipid nanoprobe to increase their binding to MDA-MB-231 cells. We 360 demonstrated that PalmReNL provides an excellent platform for characterizing the cellular uptake 361 efficiency of THP-engineered EVs by measuring bioluminescence signals. Our data further 362 indicate that PalmReNL allows for long-term tracking of individual THP-engineered EVs by 363 fluorescence microscopy.

Acknowledgments

366 This work was supported by start-up funds from MSU as well as by funding from the NIH/NINDS: R01-NS098231; R01-NS104306 NIH/NIBIB: R01-EB031008; R01-EB030565; R01-EB031936; P41-EB024495 and NSF 2027113. We would like to thank the Institute for Quantitative Health

369 Science and Engineering (IQ) at MSU for providing the facility and resources for executing this 370 work; Dr. A. Withrow at the Center for Advanced Microscopy for supporting our TEM. 
A
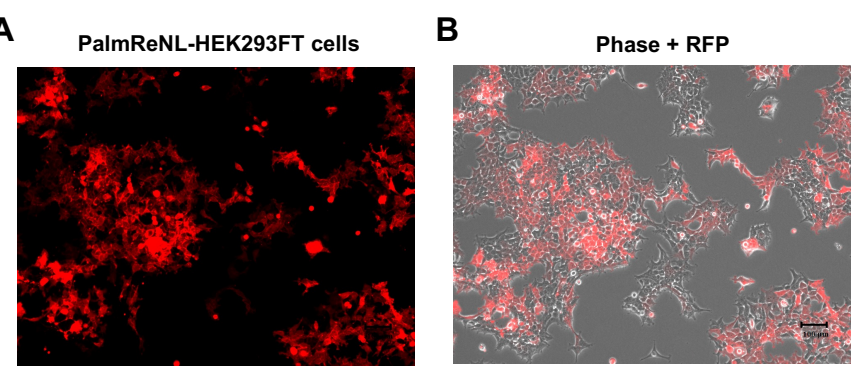

D

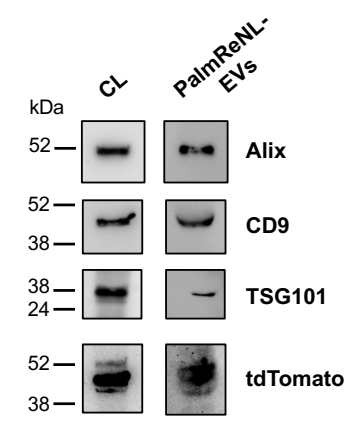

E

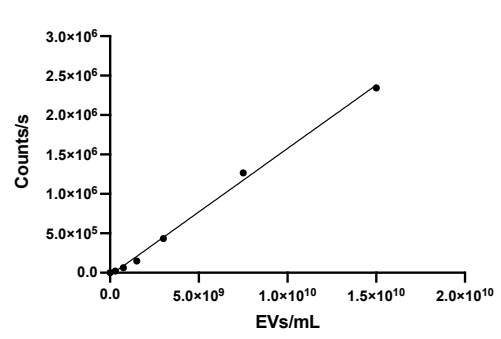

F
C
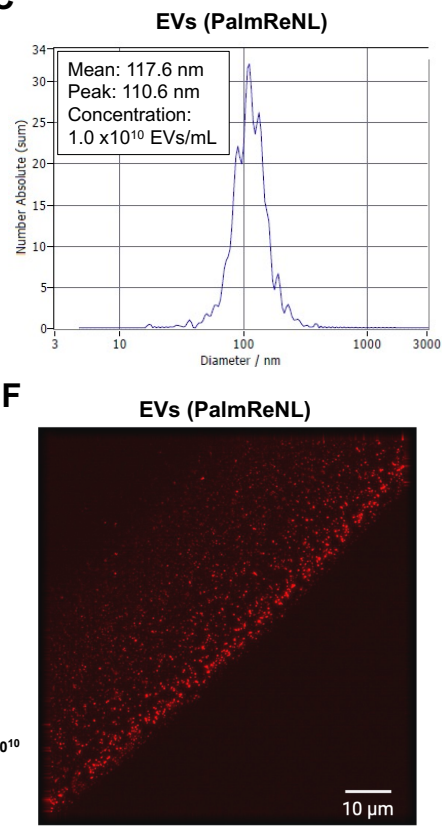

Figure 1. Characterizing dual-reporter PalmReNL-EVs. A) tdTomato fluorescence in HEK293FT cells stably expressing PalmReNL. B) A merged image of phase contrast and fluorescence microscopic images. Scale bar $=100 \mu \mathrm{m}$. C) PalmReNL-EVs were analyzed by nanoparticle tracking analysis (NTA). D) Western blot analysis of exosome marker proteins in PalmReNLHEK293FT cells and -EVs. E) Bioluminescence analysis of PalmReNL-EVs using furimazine. F) A droplet of buffer containing isolated PalmReNL-EVs visualized by fluorescence microscopy. Scale bar $=10 \mu \mathrm{m}$. 
A

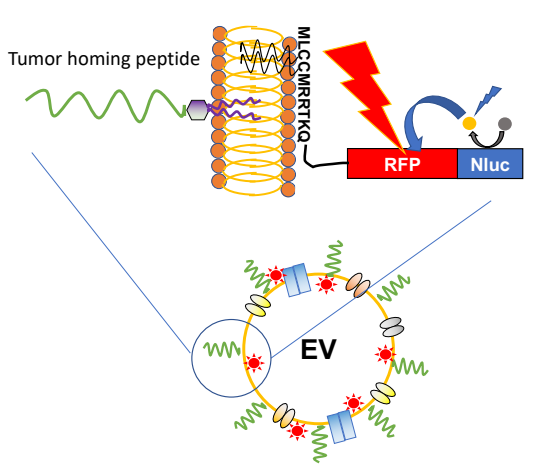

C

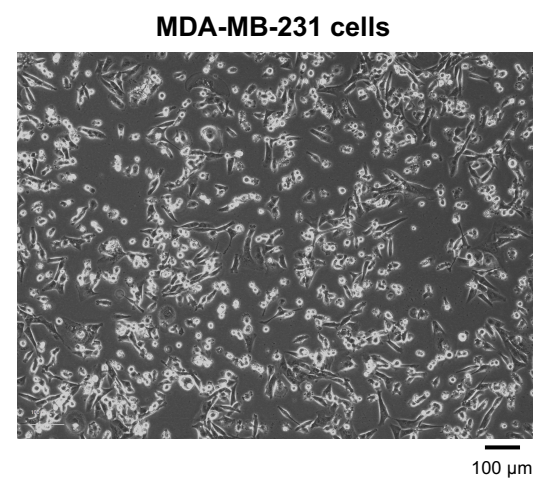

B

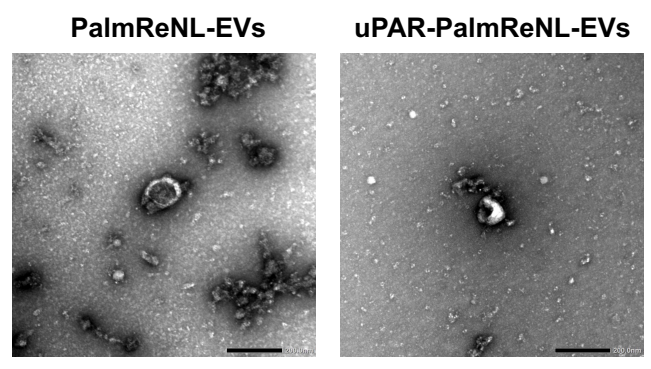

D

$\mathrm{kDa}$
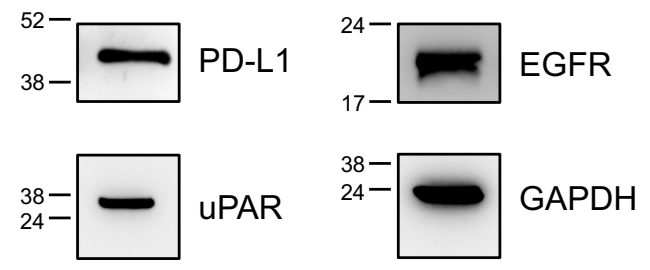

Figure 2. Engineered PalmReNL-EVs with tumor homing peptides (THPs). A) Schematic diagram of EV membrane labeling with PalmReNL BRET probes and functionalization with THP-coupled lipid nanoprobes. B) Transmission electron microscopy of HEK293FT cell-derived PalmReNLEVs with or without uPAR-binding peptides. Scale bar, $200 \mathrm{~nm}$. C) A phase contrast image of MDA-MB-231 cells. Scale bar $=100 \mu \mathrm{m}$. D) Western blot analysis of the target membrane protein expression in MDA-MB-231 cells under the non-reducing condition. GAPDH is a loading control. 
A

416

417

418

419

420

421

422

423

424

425

426

427

428

429

430

431

432
B

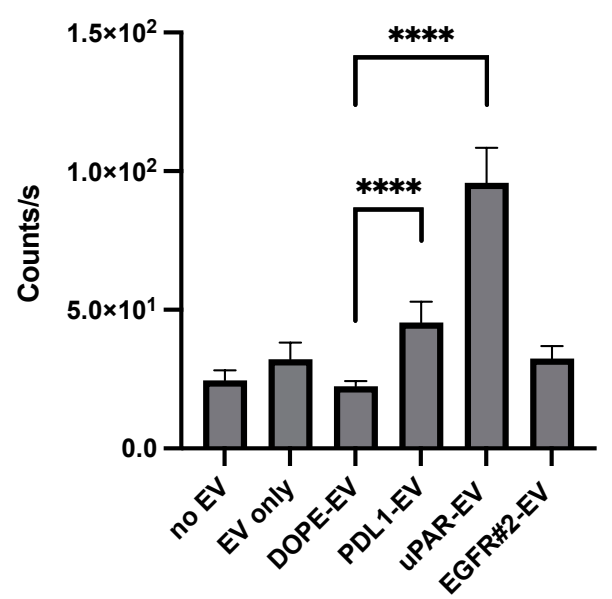

Figure 3. Bioluminescence analysis of cellular uptake of THP-engineered PalmReNL-EVs. A) Bioluminescence analysis of the recipient MDA-MB-231 cells cultured with two different concentrations of THP-engineered PalmReNL-EVs using furimazine. B) Bioluminescence analysis of cellular uptake of PalmReNL-EVs $\left(6.0 \times 10^{8} \mathrm{EVs} / \mathrm{mL}\right)$ engineered with the EGFRbinding peptide \#2, together with PalmReNL-EVs bearing PDL1-, uPAR-binding peptides, or DOPE-lipid nanoprobes only. Error bars, SD $(\mathrm{n}=5)$; two-sided Student's $t$-tests was used for comparison; **, $\mathrm{P}<0.01 ; * * *, \mathrm{P}<0.001 ; * * * *, \mathrm{P}<0.0001$. 
A

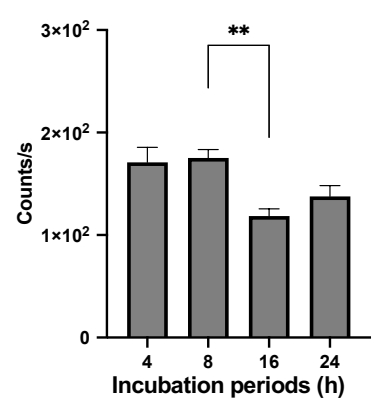

C

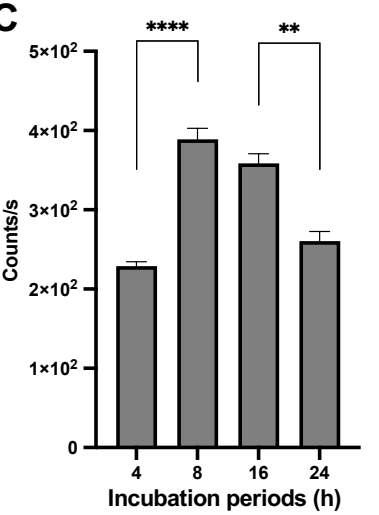

B

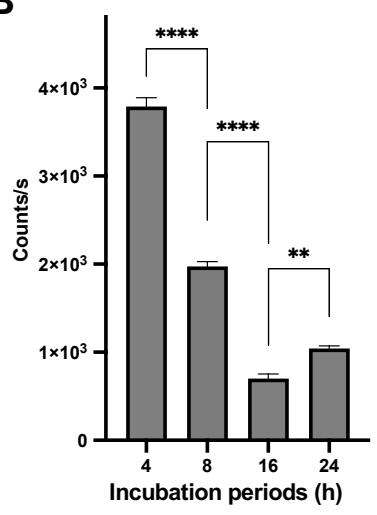

D

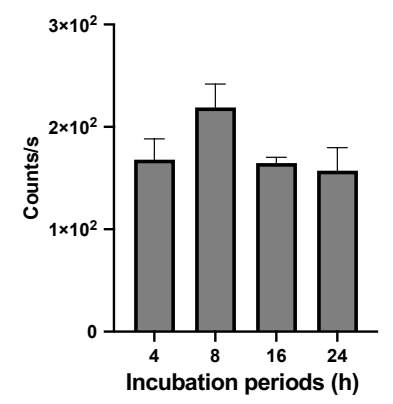

439 Figure 4. Bioluminescence analysis of time-dependent cellular uptake of THP-engineered 440 PalmReNL-EVs by MDA-MB-231 cells. Bioluminescence analysis of the recipient MDA-MB441231 cells cultured with PalmReNL-EVs bearing DOPE-lipid nanoprobes only (A), uPAR-binding 442 (B), PDL1-binding (C), or EGFR-binding (D) at 4, 8, 16, and $24 \mathrm{~h}$ post-incubation. Error bars, SD $443(\mathrm{n}=5)$; two-sided Student's $t$-test was used for comparison; **, $\mathrm{P}<0.01 ; * * * *, \mathrm{P}<0.0001$. 

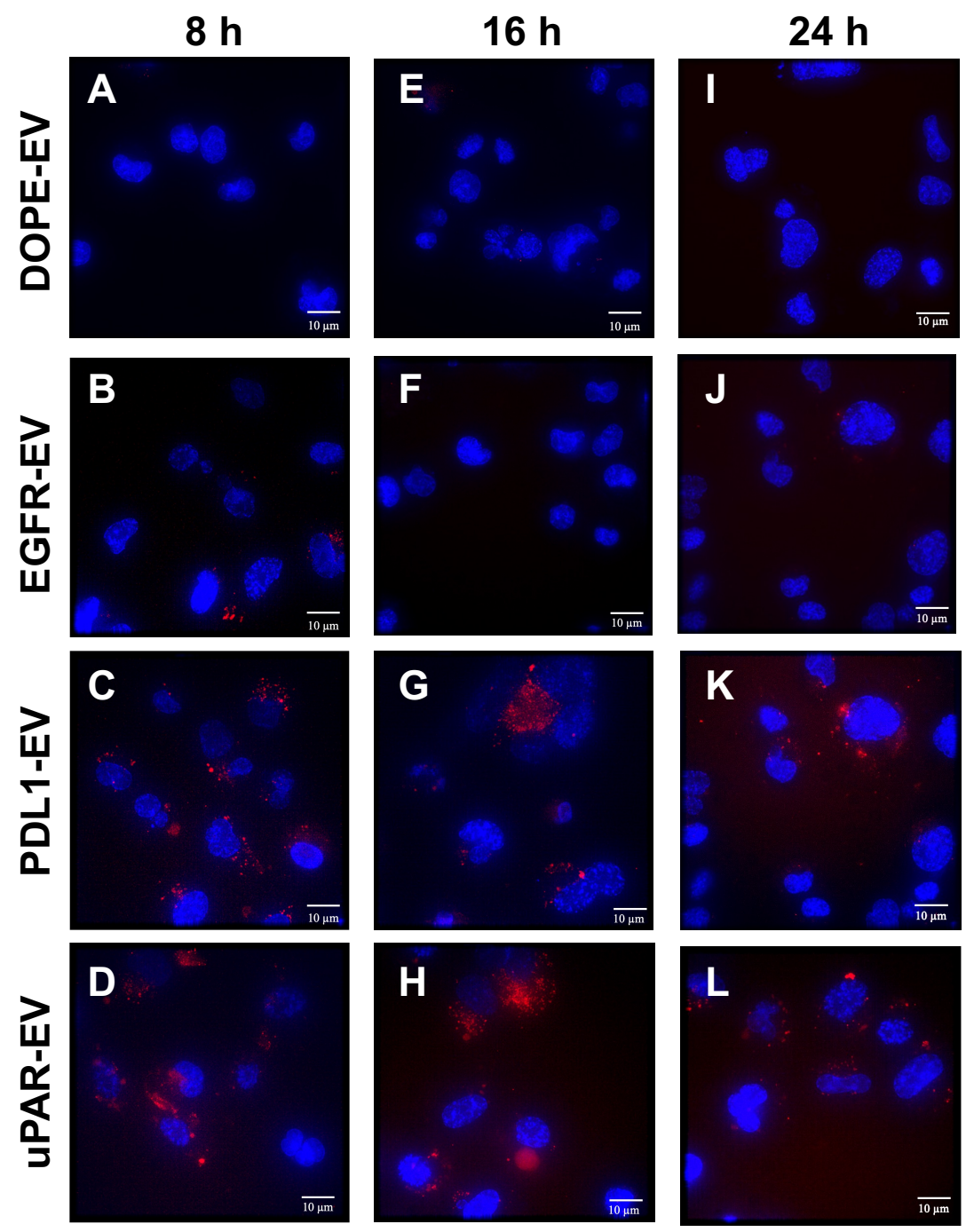

450 Figure 5. Fluorescence analysis of THP-engineered PalmReNL-EV uptake by MDA-MB-231 451 cells. MDA-MB-231 cells cultured with PalmReNL-EVs bearing DOPE-lipid nanoprobes only (A, 452 E, I), EGFR- (B, F, J), PDL1- (C, G, K), or uPAR-binding peptides (D, H, L) for 8, 16, and 24 h. 453 Punctate fluorescence signals of tdTomato (red) were merged with nuclei stained with Hoechst 45433342 (blue). Scale bar $=10 \mu \mathrm{m}$. 


\section{References}

458 1. van Niel, G., G. D'Angelo, and G. Raposo, Shedding light on the cell biology of extracellular vesicles. Nat Rev Mol Cell Biol, 2018. 19(4): p. 213-228.

2. S, E.L.A., et al., Extracellular vesicles: biolog.
Rev Drug Discov, 2013. 12(5): p. 347-57.

3. Kamerkar, S., et al., Exosomes facilitate therapeutic targeting of oncogenic KRAS in pancreatic cancer. Nature, 2017. 546(7659): p. 498-503.

4. Murphy, D.E., et al., Natural or Synthetic RNA Delivery: A Stoichiometric Comparison of Extracellular Vesicles and Synthetic Nanoparticles. Nano Lett, 2021. 21(4): p. 1888-1895.

5. Jeppesen, D.K., et al., Reassessment of Exosome Composition. Cell, 2019. 177(2): p. 428445 e18.

6. Bose, R.J.C., et al., Tumor Cell-Derived Extracellular Vesicle-Coated Nanocarriers: An

7. Yong, T., et al., Tumor exosome-based nanoparticles are efficient drug carriers for chemotherapy. Nat Commun, 2019. 10(1): p. 3838.

8. Murphy, D.E., et al., Extracellular vesicle-based therapeutics: natural versus engineered targeting and trafficking. Exp Mol Med, 2019. 51(3): p. 1-12.

9. Alvarez-Erviti, L., et al., Delivery of siRNA to the mouse brain by systemic injection of targeted exosomes. Nat Biotechnol, 2011. 29(4): p. 341-5.

10. Tian, Y., et al., A doxorubicin delivery platform using engineered natural membrane vesicle exosomes for targeted tumor therapy. Biomaterials, 2014. 35(7): p. 2383-90.

11. Ohno, S., et al., Systemically injected exosomes targeted to EGFR deliver antitumor microRNA to breast cancer cells. Mol Ther, 2013. 21(1): p. 185-91.

12. Tian, T., et al., Surface functionalized exosomes as targeted drug delivery vehicles for cerebral ischemia therapy. Biomaterials, 2018. 150: p. 137-149.

13. Pham, T.C., et al., Covalent conjugation of extracellular vesicles with peptides and nanobodies for targeted therapeutic delivery. Journal of extracellular vesicles, 2021. 10(4): p. e12057.

14. Nakase, I., et al., Vectorization of biomacromolecules into cells using extracellular vesicles with enhanced internalization induced by macropinocytosis. Sci Rep, 2016. 6: p. 34937.

15. Nakase, I., et al., Receptor clustering and activation by multivalent interaction through recognition peptides presented on exosomes. Chem Commun (Camb), 2016. 53(2): p. 317320.

16. Antes, T.J., et al., Targeting extracellular vesicles to injured tissue using membrane cloaking and surface display. J Nanobiotechnology, 2018. 16(1): p. 61.

17. Vandergriff, A., et al., Targeting regenerative exosomes to myocardial infarction using cardiac homing peptide. Theranostics, 2018. 8(7): p. 1869-1878.

18. Peinado, H., et al., Melanoma exosomes educate bone marrow progenitor cells toward a pro-metastatic phenotype through MET. Nat Med, 2012. 18(6): p. 883-91.

19. Lai, C.P., et al., Dynamic biodistribution of extracellular vesicles in vivo using a multimodal imaging reporter. ACS Nano, 2014. 8(1): p. 483-494. 
20. Takahashi, Y., et al., Visualization and in vivo tracking of the exosomes of murine melanoma B16-BL6 cells in mice after intravenous injection. J Biotechnol, 2013. 165(2): p. 77-84.

21. Gupta, D., et al., Quantification of extracellular vesicles in vitro and in vivo using sensitive bioluminescence imaging. J Extracell Vesicles, 2020. 9(1): p. 1800222.

22. Lazaro-Ibanez, E., et al., Selection of Fluorescent, Bioluminescent, and Radioactive Tracers to Accurately Reflect Extracellular Vesicle Biodistribution in Vivo. ACS Nano, 2021. 15(2): p. 3212-3227.

23. Wu, A.Y., et al., Multiresolution Imaging Using Bioluminescence Resonance Energy Transfer Identifies Distinct Biodistribution Profiles of Extracellular Vesicles and Exomeres with Redirected Tropism. Adv Sci (Weinh), 2020. 7(19): p. 2001467.

24. Schaub, F.X., et al., Fluorophore-NanoLuc BRET Reporters Enable Sensitive In Vivo Optical Imaging and Flow Cytometry for Monitoring Tumorigenesis. Cancer Res, 2015. 75(23): p. 5023-33.

25. Lai, C.P., et al., Visualization and tracking of tumour extracellular vesicle delivery and RNA translation using multiplexed reporters. Nat Commun, 2015. 6: p. 7029.

26. Shinoda, H., M. Shannon, and T. Nagai, Fluorescent Proteins for Investigating Biological Events in Acidic Environments. Int J Mol Sci, 2018. 19(6).

27. Sung, B.H., et al., A live cell reporter of exosome secretion and uptake reveals pathfinding behavior of migrating cells. Nat Commun, 2020. 11(1): p. 2092.

28. Joshi, B.S., et al., Endocytosis of Extracellular Vesicles and Release of Their Cargo from Endosomes. ACS Nano, 2020. 14(4): p. 4444-4455.

29. Zarea, A.A., et al., In vitro and in vivo analysis of microvesicle-mediated metastasis using a bright, red-shifted bioluminescent reporter protein of extracellular vesicles. bioRxiv, 2021: p. 2021.07.23.453512.

30. Suzuki, K., et al., Five colour variants of bright luminescent protein for real-time multicolour bioimaging. Nat Commun, 2016. 7: p. 13718.

31. Kapoor, P., et al., TumorHoPe: a database of tumor homing peptides. PLoS One, 2012. 7(4): p. e35187.

32. McCabe, J.B. and L.G. Berthiaume, Functional roles for fatty acylated amino-terminal domains in subcellular localization. Mol Biol Cell, 1999. 10(11): p. 3771-86.

33. Mates, L., et al., Molecular evolution of a novel hyperactive Sleeping Beauty transposase enables robust stable gene transfer in vertebrates. Nat Genet, 2009. 41(6): p. 753-61.

34. Shelke, G.V., et al., Importance of exosome depletion protocols to eliminate functional and RNA-containing extracellular vesicles from fetal bovine serum. J Extracell Vesicles, 2014. 3.

35. Liu, F., et al., The Exosome Total Isolation Chip. ACS Nano, 2017. 11(11): p. 10712-10723.

36. Kanada, M., et al., Microvesicle-Mediated Delivery of Minicircle DNA Results in Effective Gene-Directed Enzyme Prodrug Cancer Therapy. Mol Cancer Ther, 2019. 18(12): p. 23312342.

37. Devulapally, R., et al., Polymer nanoparticles mediated codelivery of antimiR-10b and antimiR-21 for achieving triple negative breast cancer therapy. ACS Nano, 2015. 9(3): p. 2290-302. 
38. Li, C., et al., Peptide Blocking of PD-1/PD-L1 Interaction for Cancer Immunotherapy. Cancer Immunol Res, 2018. 6(2): p. 178-188.

39. Liu, B., et al., Targeted theranostics of lung cancer: PD-L1-guided delivery of gold nanoprisms with chlorin e6 for enhanced imaging and photothermal/photodynamic therapy. Acta Biomater, 2020. 117: p. 361-373.

40. $\mathrm{Li}, \mathrm{Z}$., et al., Identification and characterization of a novel peptide ligand of epidermal growth factor receptor for targeted delivery of therapeutics. FASEB J, 2005. 19(14): p. 1978-85.

41. Mazzuca, C., et al., Understanding the good and poor cell targeting activity of gold nanostructures functionalized with molecular units for the epidermal growth factor receptor. Nanoscale Advances, 2019. 1(5): p. 1970-1979.

42. Hossein-Nejad-Ariani, H., E. Althagafi, and K. Kaur, Small Peptide Ligands for Targeting EGFR in Triple Negative Breast Cancer Cells. Sci Rep, 2019. 9(1): p. 2723.

43. Jung, M.K. and J.Y. Mun, Sample Preparation and Imaging of Exosomes by Transmission Electron Microscopy. J Vis Exp, 2018(131).

44. Wan, Y., et al., Rapid magnetic isolation of extracellular vesicles via lipid-based nanoprobes. Nat Biomed Eng, 2017. 1.

45. Raposo, G. and W. Stoorvogel, Extracellular vesicles: exosomes, microvesicles, and friends. J Cell Biol, 2013. 200(4): p. 373-83.

46. Yin, K.B., The mesenchymal-like phenotype of the MDA-MB-231 cell line. Breast CancerFocusing Tumor Microenvironment, Stem cells and Metastasis, 2011: p. 385-402.

47. Robers, M.B., et al., A luminescent assay for real-time measurements of receptor endocytosis in living cells. Anal Biochem, 2015. 489: p. 1-8.

48. Hall, M.P., et al., Engineered luciferase reporter from a deep sea shrimp utilizing a novel imidazopyrazinone substrate. ACS Chem Biol, 2012. 7(11): p. 1848-57.

49. Shaner, N.C., P.A. Steinbach, and R.Y. Tsien, A guide to choosing fluorescent proteins. Nat Methods, 2005. 2(12): p. 905-9.

50. Kanki, S., et al., Identification of targeting peptides for ischemic myocardium by in vivo phage display. J Mol Cell Cardiol, 2011. 50(5): p. 841-8. 